\title{
Developmental Anatomy of the Human Embryo - 3D-Imaging and Analytical Techniques
}

\author{
Shigehito Yamada1, Takashi Nakashima², Ayumi Hirose², \\ Akio Yoneyama ${ }^{3}$, Tohoru Takeda ${ }^{4}$ and Tetsuya Takakuwa ${ }^{2}$ \\ ${ }^{1}$ Congenital Anomaly Research Center, Kyoto University, \\ ${ }^{2}$ Human Health Science, Kyoto University, \\ ${ }^{3}$ Central Research Laboratory, Hitachi Ltd., \\ ${ }^{4}$ Allied Health Sciences, Kitasato University, \\ Japan
}

\section{Introduction}

Prenatal or antenatal development is a process during which the human embryo undergoes complex morphogenetic changes. To understand and characterize the dynamic events underlying human ontogenesis, it is useful to visualize embryonic structures in threedimensions (3D). Classically, solid reconstruction and fine drawing have been the primary approaches used to model the architecture of the embryonic body. The most impressive wax models of staged human embryos are housed at the Carnegie Institution of Washington DC in the Human Developmental Anatomy Center (see Fig. 1 in Chapter 1). The wax plate technique of reconstruction was first introduced to human embryology by Gustav Born (1883), and later modified in the Carnegie Laboratory in Baltimore by Osborne O. Heard and his colleagues (Heard, 1951, Heard, 1953, Heard, 1957). The procedure involves embedding of human embryos in paraffin wax, followed by serial sectioning and histological staining. Wax plates were cut faithfully as the enlarged image of each section, and the wax plates were piled up for making the 3D embryonic structures. These reconstructed models allowed for the production of accurate drawings of human embryos, some of the most notable being those of James F. Didusch, a medical artist who added valuable information to the understanding of human prenatal development (O'Rahilly, 1988). However, both solid reconstruction and fine drawings used in classical embryology are time-consuming and require specific and rare skills.

In the past decades, the visualization of biological structures has been significantly facilitated by computer-assisted techniques, allowing for the three-dimensional reconstruction of human embryos from section images and offering the unique ability to manipulate reconstructed images. The difficulties that have hindered efforts in 3D reconstruction using two-dimensional image stacks revolve around the issues of section registration and distortion. A solution has come about with the advent of Episcopic Fluorescence Image Capture (EFIC), a novel imaging modality for the generation of high- 
resolution 3D reconstruction (Weninger and Mohun, 2002; see 2.2.1). With EFIC imaging, tissue autofluorescence is used to image the block face prior to cutting each section. Although the samples have been sliced and lost during the procedure, the optical resolution of EFIC was reported to reach approximately 5-6 $\mu \mathrm{m}$ (Yamada et al., 2010) and EFIC enables us to obtain 3D images with high-resolution comparable to the images of histological serial sections. On the other hand, remarkable progress has been made in nondestructive imaging technologies such as magnetic resonance (MR) imaging. MR imaging was originally developed as a non-invasive diagnostic tool in clinical medicine, but recent technological advances has promoted its application to detailed imaging and 3D reconstruction of tiny biological structures such as embryos (Smith, 1999, 2000, 2001, Smith et al., 1994, 1996, 1999; see 2.2.2), reaching a resolution close to $30 \mu \mathrm{m} /$ pixel. X-ray technology is also widely used for non-destructive imaging of inner structures. Using the characteristics of $X$-rays as electromagnetic waves, phase-contrast $X$-ray imaging visualizes the phase-shift of $X$-ray passing through the samples and reconstructs 2D or 3D images of the samples in combination with computed tomography (Momose et al., 1996, Yoneyama et al., 2011). EFIC, MR microscopy, and phase-contrast X-ray computed tomography (CT) have all been applied to embryology. The imaging modalities are selected on the basis of their destructive vs. non-destructive features, the size of the samples and the desired resolution (Fig. 1).

Morphometrics refers to the quantitative analysis of forms, a concept that encompasses size and shape (Rohlf and Bookstein, 1990). While the qualitative morphological data obtained by classical modalities such as serial sections are not suitable for numerical conversion, the data obtained by 3D-imaging techniques (see 2.2) are easily converted for quantitative analyses. Three-dimensional morphometric analyses of human embryos from the Kyoto Collection are actively underway.

In this chapter, we will describe in detail modern modalities currently used for human embryo imaging and their applications to developmental anatomy.

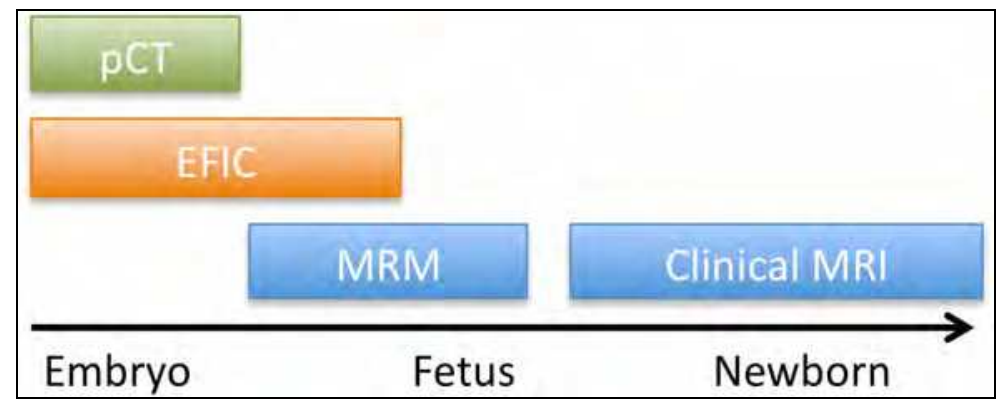

Fig. 1. Relationship between sample size and imaging techniques. pCT: phase-contrast X-ray computed tomography, EFIC: episcopic fluorescence image capture, MRM: magnetic resonance microscopy, Clinical MRI: magnetic resonance imaging for routine clinical use.

\section{Imaging modalities for three-dimensional analyses}

\subsection{Digital reconstruction from serial sections}

Classical reconstruction methods based on wax models have been described earlier. In "modern" computer-assisted reconstruction, stained histological sections are digitized using 
a digital camera equipped with a normal bright-field illumination. The color images of the serial sections (Fig. 2A) are then saved as TIFF files and a 3D reconstruction can be obtained using DeltaViewer (see 5. Appendix), a software designed to perform automated alignment and $3 \mathrm{D}$ reconstruction from serial sections. Once aligned, the images are then segmented using painting softwares (Fig. 2B) and 3D images are obtained (Fig. 2C). Further details on reconstruction procedures from serial sections can be found in previous publications (Yamada et al., 2007).
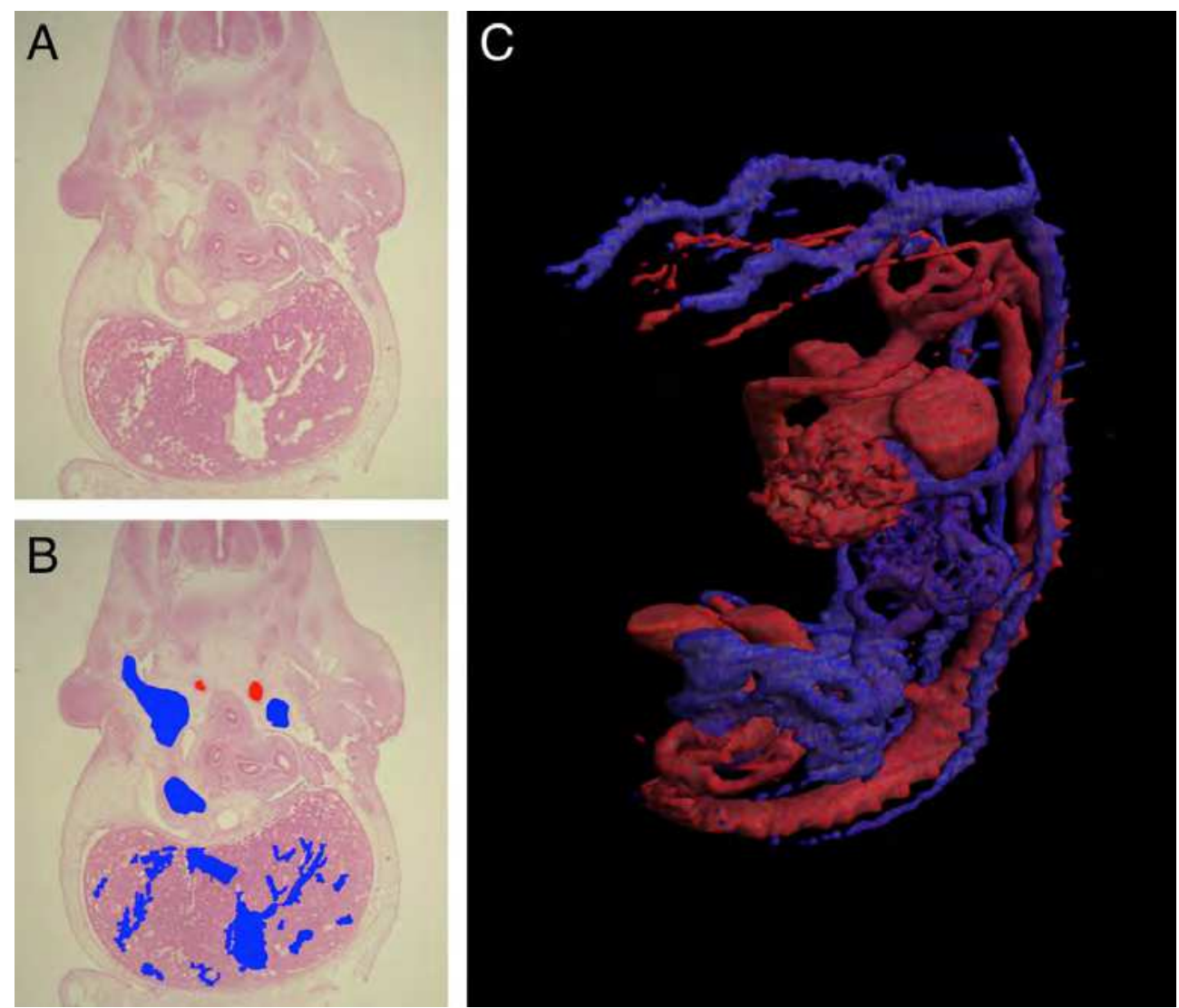

Fig. 2. Three-dimensional reconstruction from serial sections. A,B: Transverse sections of human embryo showing the spinal cord, the root of the upper limb bud, and the liver. The section is digitized (A) and manually segmented (B). C: Three-dimensional reconstruction of the heart and great vessels of human embryo at CS14 using the "DeltaViewer"software.

\subsection{D-imaging}

In contrast to serial sections, 3D-imaging allows for rapid 3D rendering such as surface reconstruction and digital resectioning in arbitrary planes. Multiple 3D-imaging modalities have been applied to the human embryos of the Kyoto Collection. 


\subsubsection{Episcopic fluorescence image capture (EFIC)}

Episcopic fluorescence image capture (EFIC) represents a novel 3D-imaging method in human embryology. This imaging technique relies on the embedding of the embryo in paraffin (Weninger and Mohun, 2002), followed by the sectioning of the block using a sliding microtome. Prior to cutting each section, the block face is imaged by capturing tissue autofluorescence. The block is accurately returned to exactly the same photo-position on the microtome, and registered 2D image stacks are automatically generated. EFIC allows for virtual resectioning of the specimen in arbitrary planes (Rosenthal et al., 2004, Weninger et al., 2006), and rapid high-resolution 3D reconstructions (Rosenthal et al., 2004). This method was applied to staged human embryos housed at the Kyoto Collection (Yamada et al., 2010; see Figure 3A).

\subsubsection{Magnetic resonance microscopy}

Magnetic resonance (MR) imaging applied to the scanning of small samples is called MR microscopy. MR microscopy is a very powerful tool for 3D measurement of chemically-fixed human embryos because of the large amounts of mobile or NMR visible protons present in the formalin preservation fluid (Matsuda et al., 2007). It is a non-invasive and nondestructive imaging process, and has been previously applied to developmental embryology in a number of animal models (Bone et al., 1986, Smith et al., 1992, 1994, 1996). MR imaging offers highly beneficial features (Effmann et al., 1988, Smith et al., 1992, Haishi et al., 2001), reaching a resolution of $40 \mu \mathrm{m} /$ pixel or higher when scanning the samples for extended periods of time. Imaging of human embryos by MR microscopy was described using superconducting magnets ranging from 1.0T to 9.4T (Smith et al., 1996, Smith et al., 1999, Haishi et al., 2001). The images shown in Fig. 3B and 3C were obtained using MR microscopes equipped with 7T and 2.34T magnets, respectively.

\subsubsection{Phase-contrast X-ray computed tomography}

X-rays are electromagnetic waves, and are thus, characterized by amplitude and phase. When an X-ray passes through a sample, its amplitude is decreased and its phase is shifted. Conventional X-ray imaging (radiography) is based on absorption-contrast (i.e. amplitude imaging) and represents the mass-density distribution of X-ray inside the sample. Its sensitivity is insufficient to perform detailed analysis of samples consisting of biological soft tissues such as embryos, unless combined with the use of contrast agents or applying higher X-ray doses. Exploiting the phase information of X-rays is a solution. The sensitivity of the phase shift for light elements such as hydrogen, carbon, nitrogen, and oxygen is about 1000 times larger than that of absorption (Momose and Fukuda, 1995). To detect a phase-shift, it is essential to convert the phase shift into a change in X-ray intensity as X-ray intensities are classically measured using current-detecting devices. Conversion methods such as interferometry and diffractometry are used for the generation of 2D and 3D observations using synchrotron radiation. Devices based on this principle have been developed (Becker and Bonse, 1974, Yoneyama et al., 2004), and an image of human embryo at CS 17 obtained using a two-crystal X-ray interferometer (Yoneyama et al., 2011) is featured in Fig. 3D. 

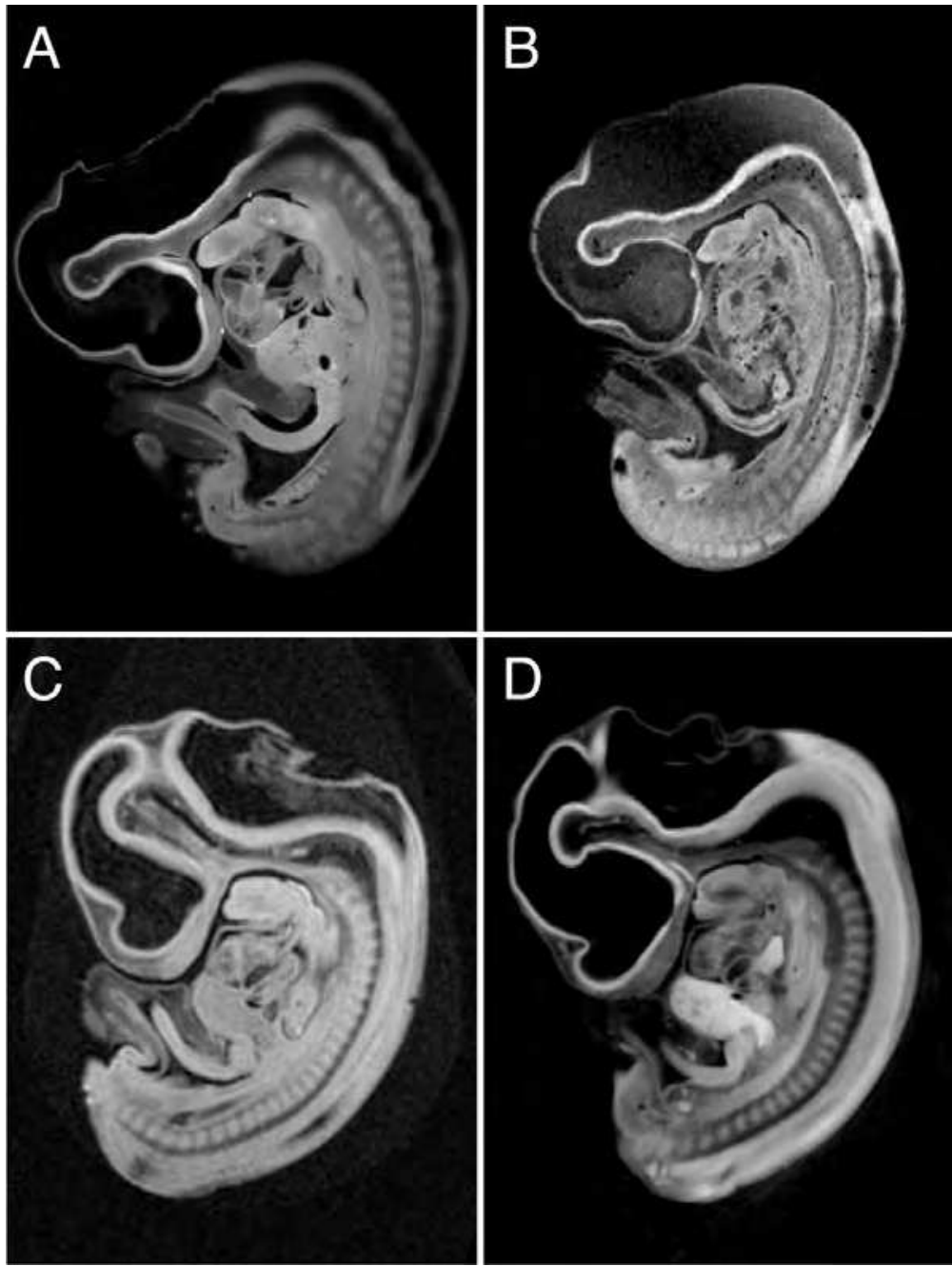

Fig. 3. Images of human embryos obtained using various imaging modalities. CS16 embryo imaging using EFIC (A) and 7T-MRI (B), CS17 embryo imaging using 2.34T MRI (C) and phase-contrast $\mathrm{x}$-ray $\mathrm{CT}$ at $17.8 \mathrm{keV} \mathrm{X}$-ray energy (D).

\section{Analyses of developmental anatomy using 3D-imaging}

\subsection{MR microscopy project at the Kyoto collection of human embryos}

The Kyoto collection counts approximately 45,000 human embryos, and contains historical specimens housed at the Congenital Anomaly Research Center of Kyoto University (Nishimura et al., 1968, Nishimura, 1975, Shiota, 1991, Yamada et al., 2004). Most specimens were obtained from pregnancies terminated during the first trimester due to socioeconomic reasons as legally permitted under the Maternity Protection Law of Japan. Some of the 
specimens $(\sim 20 \%)$ are undamaged, well-preserved embryos. When the aborted materials were brought to the laboratory, the embryos were measured, examined, and staged according to the criteria of O'Rahilly and Müller (1987). Further information on the Kyoto Collection of Human Embryos can be found in Chapter 1. In 1999, Kyoto University and the University of Tsukuba initiated a collaborative project aiming to acquire 3D MR microscopic images of thousands of human embryos using a super-parallel MR microscope operated at 2.34T (Matsuda et al., 2003, 2007, Yamada et al., 2006, Shiota et al., 2007). During the course of the project, over 1,200 human embryos were scanned. Further information on the data generated can be found on the web (http://mrlab.frsc.tsukuba.ac.jp/human_embryos/).

\subsection{Flow chart: from MR image acquisition to 3D image reconstruction}

Approximately 1,200 well-preserved human embryos diagnosed as externally normal at CS13 to CS23 were selected for MR microscopic imaging (Fig. 4A)(Matsuda et al., 2003, 2007,

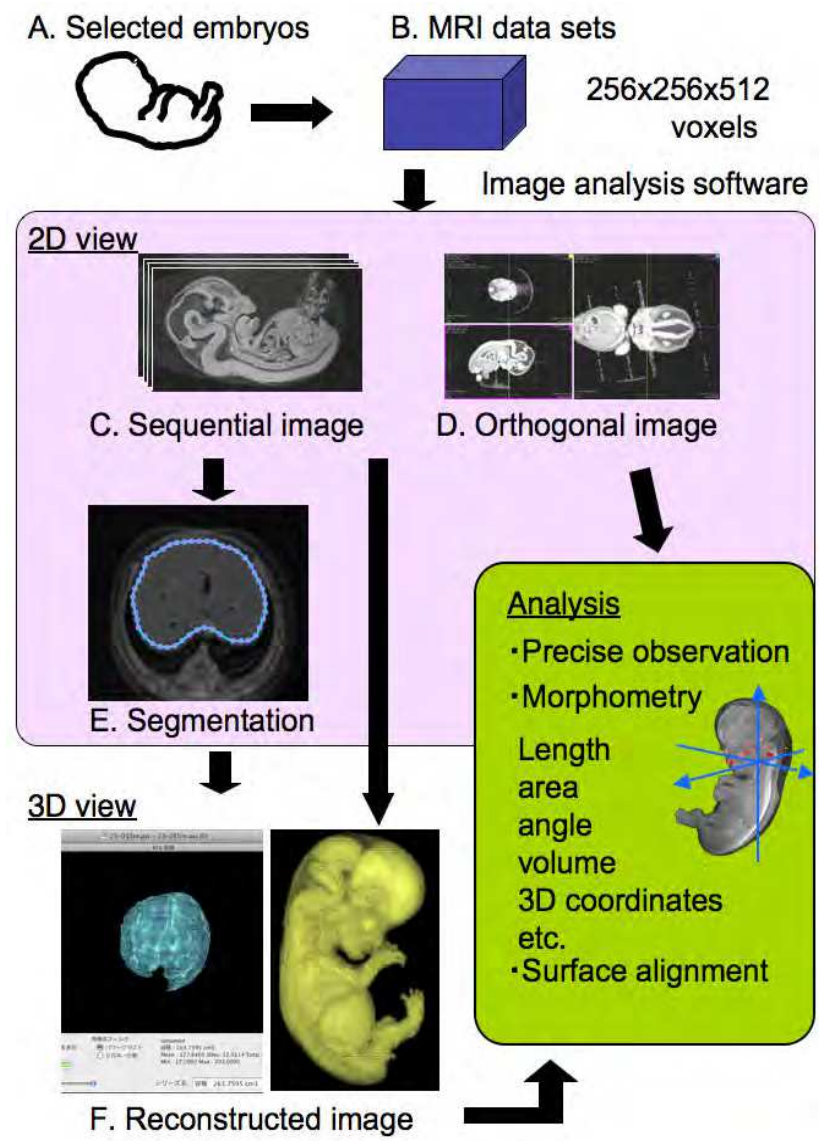

Fig. 4. Flow chart: from MR image acquisition to 3D image reconstruction 

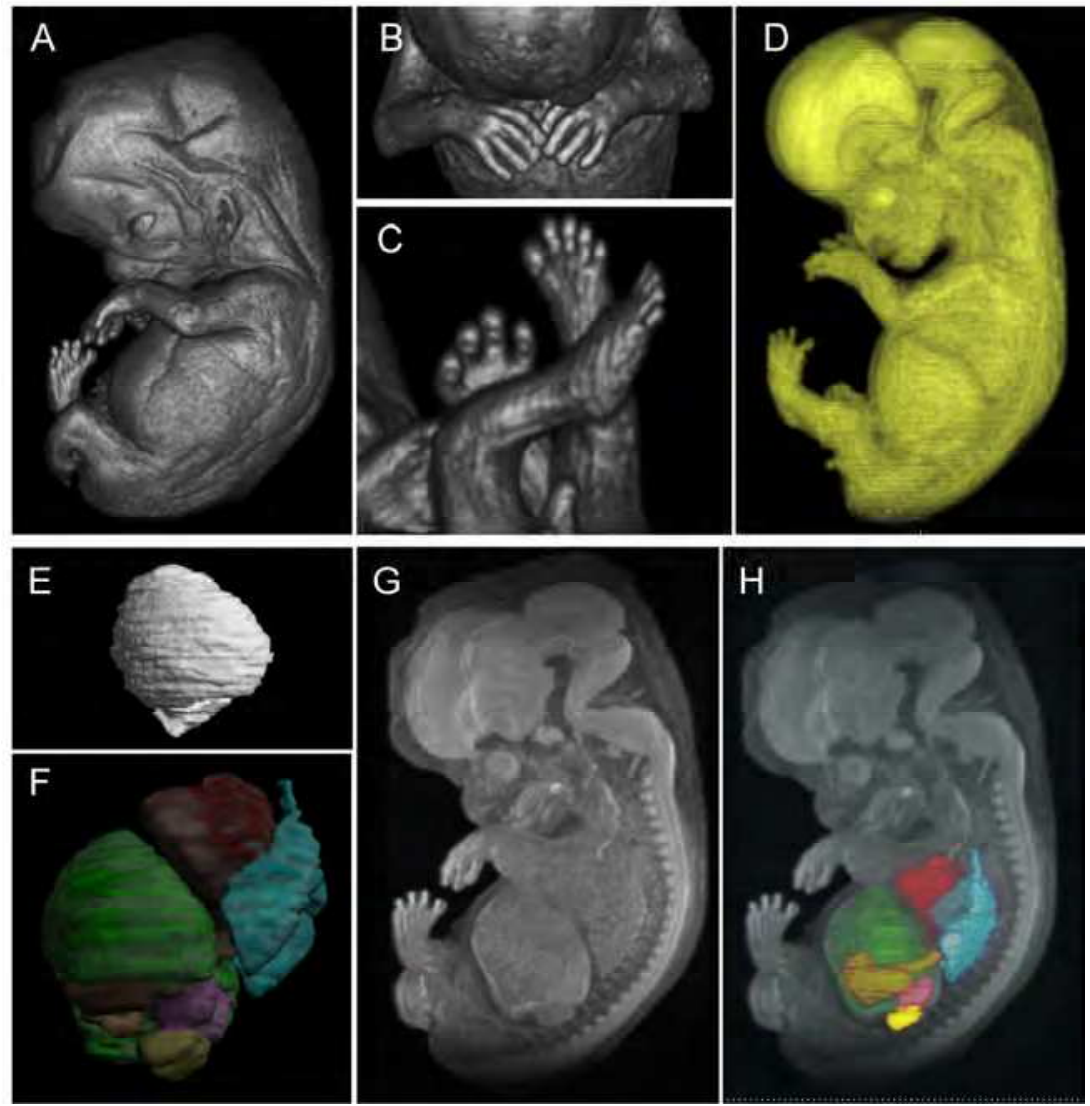

Fig. 5. Samples of 3D reconstructed images. A) 3D images of whole embryo at CS 23 using volume rendering algorithm (Osirix) to observe surfaces. B,C) Magnification of upper and lower extremities, demonstrating fine and detailed reconstruction of embryonic morphology. D) When modifying the volume-rendering settings, both external and internal embryonic structures can be observed. E) 3D-reconstruction of the embryonic liver at CS 23 obtained from segmentation of 2D sequential images. F) Liver (green), lung (blue), heart (red), kidney (yellow), and adrenal glands (purple) were segmented from 2D sequential images and reconstructed in 3D. G) 3D-reconstruction using Maximum intensity projection (MIP) tool (Osirix) in order to generate both surface and internal imaging perspectives. $\mathrm{H}$ ) Organ images shown in F were overlaid with MIP images shown in G.

Yamada et al., 2006, Shiota et al., 2007). The 3D MR image datasets for each embryo were initially obtained from 256x256x512 voxels (Fig. 4B). Each dataset was subsequently converted into two-dimensional (2D) image stacks (Fig. 4C), which were then digitally resectioned following predefined planes (Fig. 4D). Organs of interest were segmented in series of 2D images (Fig. 4E) and the 3D architectures were computationally reconstructed (Fig. 4F). Images obtained can be freely rotated on the screen, and 3D shapes are easily recognizable and their spatial relationships rapidly determined. The obtained 2D and 3D images obtained can be subjected to further analysis. 


\subsection{Further processing of reconstructed 3D images using computer software}

Recent advances in computer technology have significantly facilitated image rendering on personal computers. A number of algorithms have been developed resulting in multiple 3D reconstruction softwares, many of which are available as open-source. The most popular softwares are summarized in the Appendix section of this chapter. Samples of reconstructed images using such rendering algorithms are represented in Fig. 5.

\subsubsection{Imaging using volume rendering techniques (Fig. 5A-D)}

Volume rendering techniques are utilized to reconstruct whole embryo images. The display and comparative analysis of 3D images at various developmental stages enables a clearer understanding of embryonic morphogenesis.

Carnegie stages are primarily defined based on external structural features, e.g. cranial facial morphogenesis including eye, nose, pharyngeal arches related organs, posture of the whole embryo, finger and toe development (O'Rahilly and Müller, 1987). The external morphologies obtained by volume rendering have enough quality to determine the developmental stages of the embryos. Because these external morphologies are strictly preserved in this method, the judgment of the staging was identical with that used with original embryo specimens.

\subsubsection{Three-dimensional reconstruction from segmentation of 2D sequential images} (Fig. 5E, 5F)

Regions of interest (ROI) were segmented from 2D images and then reconstructed using ROI and Osirix reconstruction module. Multiple organs can be individually segmented and combined into 3D images, showing a spatial relationship clearly between adjacent organs. One limitation to 3D image rendering is the lack of information on color and touch sense e.g. pigmentation of the retina, color of superficial arteries or internal organs such as the heart. When modifying the volume rendering settings, external and internal embryonic structures can be observed simultaneously.

\subsubsection{Imaging using maximum intensity projection (MIP) method (Fig. 5G, 5H)}

Information on both external and internal structures can be acquired using the MIP method (Nakashima et al., 2011). Three-dimensional images obtained by MIP can be superimposed with 3D reconstruction obtained from segmented images, thus creating a see-through effect with internal organs visible from the outside.

\subsection{Analysis on 3D reconstructed images}

\subsubsection{Three-dimensional morphological observation}

Three-dimensional reconstruction offers a number of advantages. For instance, the resulting image is amenable to comprehensive examination as the image can be freely rotated on the screen, 3D shapes are easily recognizable and spatial relationships between adjacent organs or tissues become obvious. In Fig. 6, 3D reconstructed images of the embryonic liver (CS18) 
represented with (Fig. 6A) and without (Fig. 6B) the adjacent heart can be compared and reveal the anatomic relationship between the two organs. Indeed, the recess formed by the left ventricle is a characteristic temporal feature of the cranial surface of the liver between CS17 and CS19.
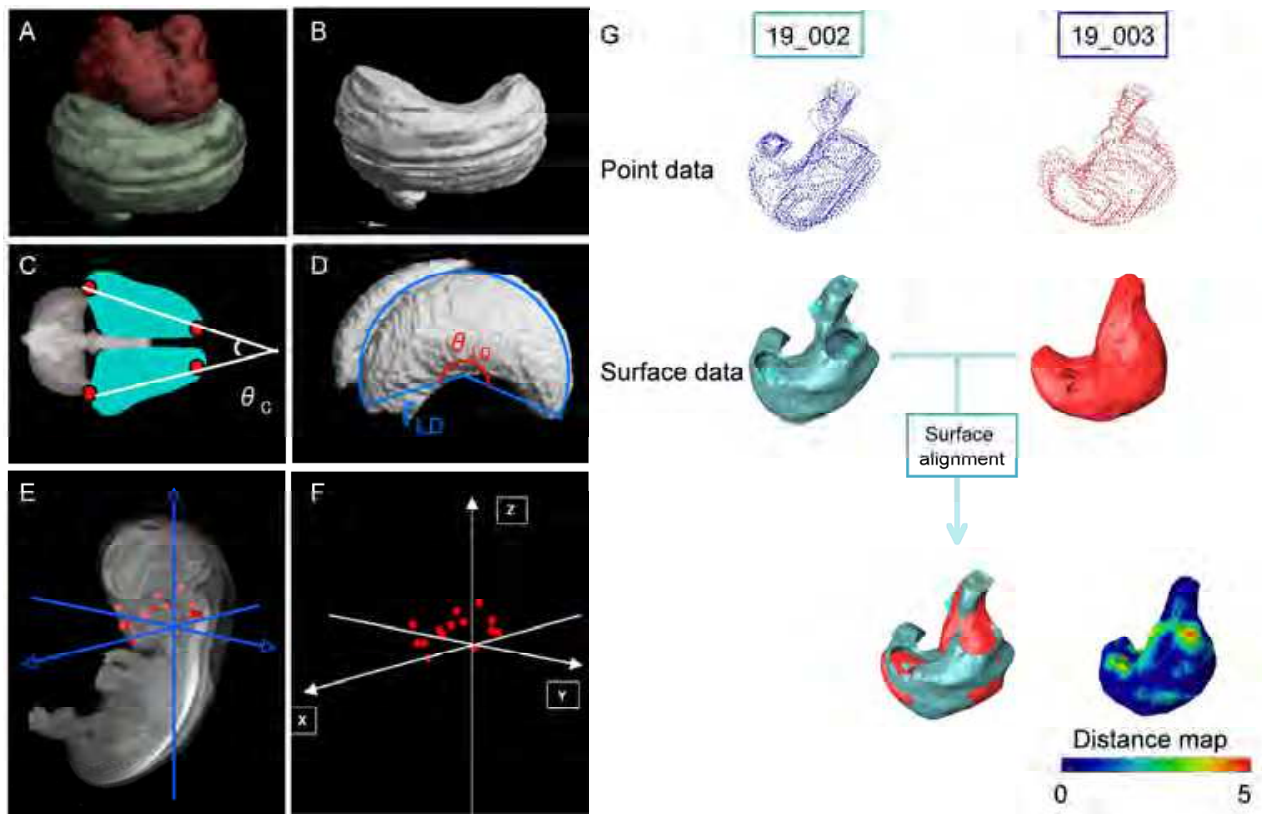

Fig. 6. Analytical methods on 3D reconstructed images.

A,B) Three-dimensional reconstructed image of the embryonic liver (CS18) with (A) and without (B) the heart demonstrate the anatomical relationship between the two organs. C-D) Morphometry from 3D images of lateral cerebral ventricles (CS22): C) Cranial view. The blue shaded areas represent the lateral ventricles and the angle formed by the bilateral ventricles was measured. D) Lateral view. The viewing perspective was modified allowing measurement of radius and central angles.

E,F) Three-dimensional coordinates of anatomical landmarks are useful for monitoring movements between developmental stages and characterize relationships between anatomical landmarks.

G) Surface alignment provides an averaged view of the organ of interest. Here, the stomachs from two embryos at CS19 were aligned.

\subsubsection{Morphometry}

Three-dimensional images can be exploited to measure morphological changes in a quantitative manner. Using the image data, not only the total volumes, but also the lengths, angles and areas of the regions and organs of interest can be measured accurately (Fig. 6C, 6D). Morphometric data are useful for evaluating and characterizing developmental features of the embryo, and also for screening for abnormalities. 


\subsubsection{Three-dimensional coordinates}

MRI data sets are provided as cuboid of 256x 256x512 voxels and thus allows for threedimensional coordinates to be assigned to embryonic landmarks (Fig. 6E, 6F). Threedimensional coordinates of anatomical landmarks are useful for monitoring the movements of landmarks and define their anatomical relationships during prenatal development.

\subsubsection{Surface alignment}

Multiple images can be aligned resulting in averaged images and compatibility rates are indicated by color gradients. An embryonic stomach was segmented from 2D sequential images (Fig. 6G) and surface data originating from point datasets of each respective embryo were processed.


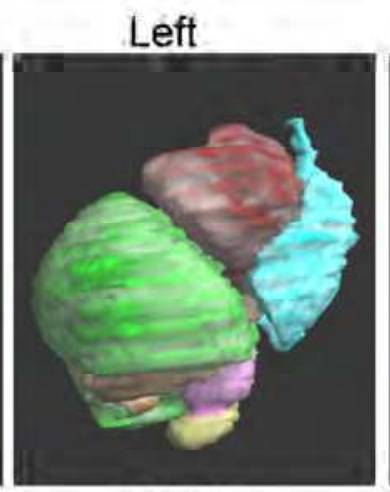

Right

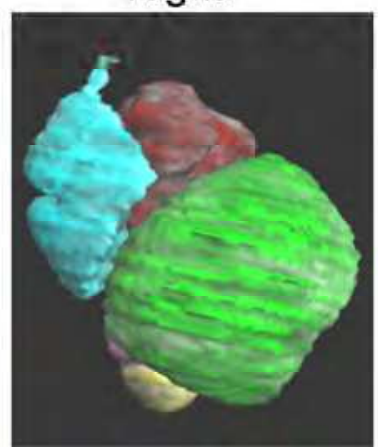

\section{Cranial}

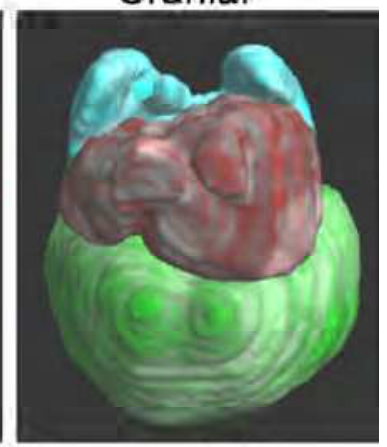

Caudal

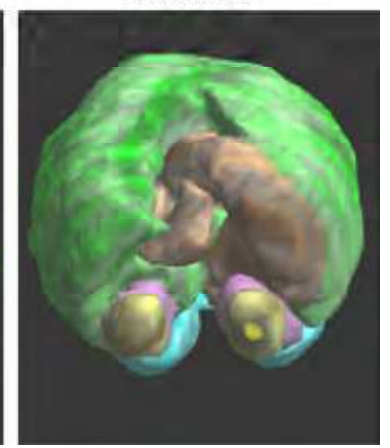

Fig. 7. Representative 3D images of the embryonic liver at CS22 with adjacent organs. Liver (green), lung (blue), heart (red), stomach (brown), kidney (yellow), and adrenal glands (purple) were segmented from 2D sequential images and reconstructed in 3D.

\subsection{Representative 3D images of the embryo and fetus organs}

Three-dimensional images of various organs in the embryo or the fetus were constructed and representative images of embryonic liver and cerebral ventricles are shown in Fig. 7 and Fig. 8, respectively. 


\section{Perspective}

Recent advances in imaging techniques allow for anatomical analyses of human embryo specimens in earlier stages and for clinical prenatal diagnosis during the first trimester. Current information on normal development during embryonic stages, however, remains insufficient to achieve such clinical evaluation. Further investigations are critical to gain insight into the dynamic and complex events occurring during organogenesis. Dynamic modeling of embryonic structures and 3D digital reconstructions will be valuable tools to elucidate the complex anatomical changes taking place during early embryonic stages. They will serve as useful references to evaluate the appropriate development of embryonic organs, and understand how adjacent organs affect each other's morphology. Now and in the future, this type of information will be indispensable to researchers and to clinicians, and more particularly in respect to the obstetrical ultrasonography conducted in the early gestational weeks.

\section{Appendix (softwares)}

The use of software is necessary for reconstruction into 3D images and morphometric analysis. The software programs used in this chapter are summarized below. More information is available on the URL of their respective websites.

\subsection{OsiriX (http://www.osirix-viewer.com/index.html)}

OsiriX is an image processing software dedicated to DICOM images produced by imaging equipment (e.g. MRI, CT, PET, PET-CT, SPECT-CT, Ultrasounds). It is fully compliant with the DICOM standard for image communication and image file formats. OsiriX is able to receive images transferred by DICOM communication protocol from any PACS or imaging modality.

\subsection{Image J (http://rsbweb.nih.gov/ij/index.html)}

ImageJ is a public domain Java image-processing program inspired from the NIH Image software developed for Macintosh. It runs, either as an online applet or as a downloadable application, on any computer with a Java 1.4 or later virtual machine.

\subsection{Delta viewer (http://delta.math.sci.osaka-u.ac.jp/DeltaViewer/index.html)}

DeltaViewer is an application program developed for Apple Macintosh. DeltaViewer reads sequences of cross-sectional images of a sample in a manner similar to confocal laser microscopes, CT, MRI, optical or electron microscopes. The computer program then reconstructs the surface of the scanned sample, and displays the image on the screen. The image can then be freely rotated, for characterization of 3D shapes and spatial relationships.

\subsection{Avizo (http://www.vsg3d.com/avizo/overview)}

Avizo ${ }^{\circ}$ software is a powerful, multifaceted tool for visualizing, manipulating, and understanding scientific and industrial data. Wherever 3D data sets need to be processed, in materials science, geosciences, environmental or engineering applications, Avizo offers abundant state-of-the-art features within an intuitive workflow and easy-to-use graphical user interfaces. 

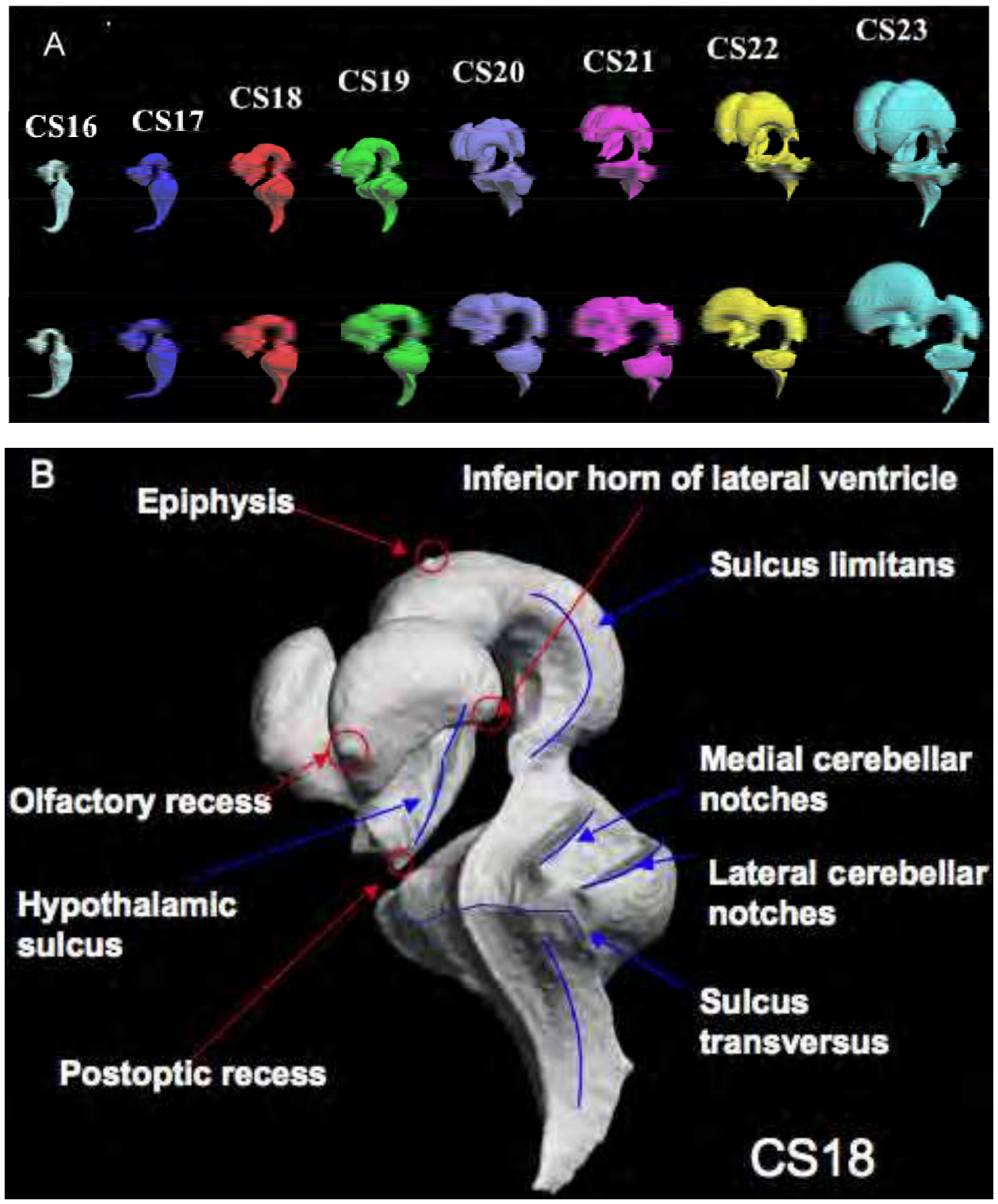

Fig. 8. (A) Representative 3D images of cerebral ventricles between Carnegie stage 16 and stage 23. (B) 3D image illustrating the conservation of anatomic landmarks.

\subsection{FMRIB Software Library (FSL) (http://www.fmrib.ox.ac.uk/fsl/index.html)}

FSL is a comprehensive library of analytical tools for fMRI (functional magnetic resonance imaging), MRI and DTI (Diffusion tensor imaging) brain imaging data. FSL was mainly 
developed by members of the Analysis Group at the FMRIB, Oxford, UK. FSL runs on Apple and PCs (Linux and Windows), and is easy to install. Most of the tools can be run either from the command line or as "point-and-click" graphical user interfaces.

\subsection{Analyze (http://www.mayo.edu/bir/Software/Analyze/Analyze.html)}

Analyze 10.0 is a powerful, comprehensive software package for multi-dimensional display, processing, and measurement of multi-modality biomedical images. Product of more than 25 years of biomedical imaging research and development at Mayo Clinic, this integrated, total solution allows you to significantly enhance your multidimensional biomedical imaging productivity.

\section{Acknowledgments}

We would like to thank Ms Merumo Ueda, Ms Nami Uematsu, Ms Kyoko Nakajima, and Ms Sayuri Nunomura at the Kyoto University Graduate School of Medicine, Human Health Science, for conducting some of the experiments; Ms Chigako Uwabe at the Congenital Anomaly Research Center for technical assistance in handling human embryos; Prof. Masaaki Wada at the Graduate School of Information Science and Technology at Osaka University for help on the use of the DeltaViewer software; Prof. Katsumi Kose and Dr. Yoshimasa Matsuda at the Institute of Applied Physics at University of Tsukuba and Dr. Stasia A Anderson at the NHLBI Animal MRI Core, National Institutes of Health, for technical help with MR imaging; and Prof. Kohei Shiota, Vice President of Kyoto University, for his support and guidance on the project. The researches were financially supported by Grants \#228073, \#238058, \#21790810 and \#22591199 from the Japan Society for the Promotion of Science (JSPS) and the Japan Science and Technology (JST) institute for Bioinformatics Research and Development (BIRD). The researches were also supported by Japan Spina Bifida and Hydrocephalus Research Foundation, and Konica Minolta Science and Technology Foundation. The studies presented in this chapter were approved by the Medical Ethics Committee at Kyoto University Graduate School of Medicine (Kyoto, Japan).

\section{References}

Becker, B. P. \& Bonse, U. 1974. The skew-symmetric two-crystal X-ray interferometer. Journal of Applied Crystallography, 7, 593-598.

Bone, S. N., Johnson, G. A. \& Thompson, M. B. 1986. Three-dimensional magnetic resonance microscopy of the developing chick embryo. Invest Radiol, 21, 782-7.

Born, G. 1883. Die Plattenmodelliermethode. Archiv für mikroskopische Anatomie. 22, 584-99.

Effmann, E. L., Johnson, G. A., Smith, B. R., Talbott, G. A. \& Cofer, G. 1988. Magnetic resonance microscopy of chick embryos in ovo. Teratology, 38, 59-65.

Haishi, T., Uematsu, T., Matsuda, Y. \& Kose, K. 2001. Development of a 1.0 T MR microscope using a Nd-Fe-B permanent magnet. Magnetic resonance imaging, 19, 875-80.

Heard, O. O. 1951. Section compression photographically rectified. The Anatomical record, $109,745-55$. 
Heard, O. O. 1953. The influence of surface forces in microtomy. The Anatomical record, 117, 725-39.

Heard, O. O. 1957. Methods used by C.H. Heuser in preparing and sectioning early embryos. Contributions to Embryology, 36, 1-18.

Matsuda, Y., Ono, S., Otake, Y., Handa, S., Kose, K., Haishi, T., Yamada, S., Uwabe, C. \& Shiota, K. 2007. Imaging of a large collection of human embryo using a superparallel MR microscope. Magnetic resonance in medical sciences : MRMS : an official journal of Japan Society of Magnetic Resonance. 6, 139-46.

Matsuda, Y., Utsuzawa, S., Kurimoto, T., Haishi, T., Yamazaki, Y., Kose, K., Anno, I. \& Marutani, M. 2003. Super-parallel MR microscope. Magnetic resonance in medicine : official journal of the Society of Magnetic Resonance in Medicine / Society of Magnetic Resonance in Medicine. 50, 183-9.

Momose, A. \& Fukuda, J. 1995. Phase-contrast radiographs of nonstained rat cerebellar specimen. Medical physics, 22, 375-9.

Momose, A., Takeda, T., Itai, Y. \& Hirano, K. 1996. Phase-contrast X-ray computed tomography for observing biological soft tissues. Nature medicine. 2, 473-5.

Nakashima, T., Hirose, A., Yamada, S., Uwabe, C., Kose, K. \& Takakuwa, T. 2011. Morphometric analysis of the brain vesicles during the human embryonic period by magnetic resonance microscopic imaging. Congenital Anomalies. doi: 10.1111/j.1741-4520.2011.00345.x

Nishimura, H. 1975. Prenatal versus postnatal malformations based on the Japanese experience on induced abortions in the human being. . In: BLANDEU, R. (ed.) Aging Gamates. Basel: S. Karger AG.

Nishimura, H., Takano, K., Tanimura, T. \& Yasuda, M. 1968. Normal and abnormal development of human embryos: first report of the analysis of 1,213 intact embryos. Teratology, 1, 281-90.

O'Rahilly, R. 1988. One Hundred Years of Human Embryology. In: KALTER, H. (ed.) Issues and Reviews in Terratology New York: Plenum Press.

O'Rahilly, R. \& Müller, F. 1987. Developmental stages in human embryos: including a revision of Streeter's "horizons" and a survey of the Carnegie Collection., Washington, DC, Carnegie Institution of Washington Publication.

Rohlf, F. J. \& Bookstein, F. L. 1990. Proceedings Of The Michigan Morphometrics Workshop, Ann Arbor, MI, University of Michigan Museum of Zoology.

Rosenthal, J., Mangal, V., Walker, D., Bennett, M., Mohun, T. J. \& Lo, C. W. 2004. Rapid high resolution three dimensional reconstruction of embryos with episcopic fluorescence image capture. Birth defects research. Part C, Embryo today : reviews, 72, 213-23.

Shiota, K. 1991. Development and intrauterine fate of normal and abnormal human conceptuses. Congenital Anomalies, 31, 67-80.

Shiota, K., Yamada, S., Nakatsu-Komatsu, T., Uwabe, C., Kose, K., Matsuda, Y., Haishi, T., Mizuta, S. \& Matsuda, T. 2007. Visualization of human prenatal development by magnetic resonance imaging (MRI). American journal of medical genetics. Part A, 143A, 3121-6.

Smith, B. R. 1999. Visualizing human embryos. Scientific American, 280, 76-81.

Smith, B. R. 2000. Magnetic resonance imaging analysis of embryos. Methods in molecular biology, 135, 211-6. 
Smith, B. R. 2001. Magnetic resonance microscopy in cardiac development. Microscopy research and technique, 52, 323-30.

Smith, B. R., Effmann, E. L. \& Johnson, G. A. 1992. MR microscopy of chick embryo vasculature. Journal of magnetic resonance imaging: JMRI. 2, 237-40.

Smith, B. R., Huff, D. S. \& Johnson, G. A. 1999. Magnetic resonance imaging of embryos: an Internet resource for the study of embryonic development. Computerized medical imaging and graphics : the official journal of the Computerized Medical Imaging Society, 23, 33-40.

Smith, B. R., Johnson, G. A., Groman, E. V. \& Linney, E. 1994. Magnetic resonance microscopy of mouse embryos. Proc Natl Acad Sci U S A, 91, 3530-3.

Smith, B. R., Linney, E., Huff, D. S. \& Johnson, G. A. 1996. Magnetic resonance microscopy of embryos. Computerized medical imaging and graphics: the official journal of the Computerized Medical Imaging Society. 20, 483-90.

Weninger, W. J., Geyer, S. H., Mohun, T. J., Rasskin-Gutman, D., Matsui, T., Ribeiro, I., Costa Lda, F., Izpisua-Belmonte, J. C. \& Muller, G. B. 2006. High-resolution episcopic microscopy: a rapid technique for high detailed $3 \mathrm{D}$ analysis of gene activity in the context of tissue architecture and morphology. Anatomy and embryology, 211, 213-21.

Weninger, W. J. \& Mohun, T. 2002. Phenotyping transgenic embryos: a rapid 3-D screening method based on episcopic fluorescence image capturing. Nature genetics, 30, 59-65.

Yamada, S., Itoh, H., Uwabe, C., Fujihara, S., Nishibori, C., Wada, M., Fujii, S. \& Shiota, K. 2007. Computerized three-dimensional analysis of the heart and great vessels in normal and holoprosencephalic human embryos. Anatomical record : advances in integrative anatomy and evolutionary biology, 290, 259-67.

Yamada, S., Samtani, R. R., Lee, E. S., Lockett, E., Uwabe, C., Shiota, K., Anderson, S. A. \& Lo, C. W. 2010. Developmental atlas of the early first trimester human embryo. Developmental dynamics : an official publication of the American Association of Anatomists, 239, 1585-95.

Yamada, S., Uwabe, C., Fujii, S. \& Shiota, K. 2004. Phenotypic variability in human embryonic holoprosencephaly in the Kyoto Collection. Birth Defects Res A Clin Mol Teratol, 70, 495-508.

Yamada, S., Uwabe, C., Nakatsu-Komatsu, T., Minekura, Y., Iwakura, M., Motoki, T., Nishimiya, K., Iiyama, M., Kakusho, K., Minoh, M., Mizuta, S., Matsuda, T., Matsuda, Y., Haishi, T., Kose, K., Fujii, S. \& Shiota, K. 2006. Graphic and movie illustrations of human prenatal development and their application to embryological education based on the human embryo specimens in the Kyoto collection. Developmental dynamics : an official publication of the American Association of Anatomists, 235, 468-77.

Yoneyama, A., Takeda, T., Tsuchiya, Y., Wu, J., Lwin, T. T., Koizumi, A., Hyodo, K. \& Itai, Y. 2004. A phase-contrast X-ray imaging system - with a $60 \times 30 \mathrm{~mm}$ field of viewbased on a skew-symmetric two-crystal X-ray inteferometer. Nuclear Instruments and Methods in Physics Research Section A: Accelerators, Spectrometers, Detectors and Associated Equipment, 523, 217-222. 
Yoneyama, A., Yamada, S. \& Takeda, T. 2011. Fine Biomedical Imaging Using X-Ray PhaseSensitive Technique. In: Gargiulo, D. G., Mcewan, A. (ed.) Advanced Biomedical Engineering. InTech. p107-128. 


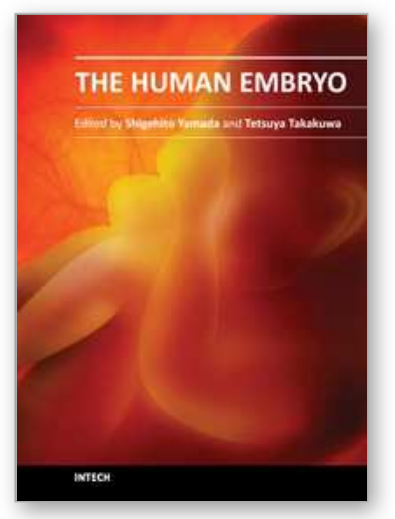

\author{
The Human Embryo \\ Edited by Dr. Shigehito Yamada
}

ISBN 978-953-51-0124-6

Hard cover, 180 pages

Publisher InTech

Published online 02, March, 2012

Published in print edition March, 2012

Human embryology is now rapidly moving to a new phase due to recent innovation and advances of life science including ES and iPS technology. This new era also directs a difficult challenge for scientists in terms of technological and ethical issues for future human embryology. However, human embryology is difficult to research due to ethics involved in the collection of human materials. This book traces the early history and provides knowledge on demonstration of principles from ancient to the most recent embryo studies amidst the unresolved scientific and ethical issues. We hope this book will help the readers to understand human embryo development better.

\title{
How to reference
}

In order to correctly reference this scholarly work, feel free to copy and paste the following:

Shigehito Yamada, Takashi Nakashima, Ayumi Hirose, Akio Yoneyama, Tohoru Takeda and Tetsuya Takakuwa (2012). Developmental Anatomy of the Human Embryo - 3D-Imaging and Analytical Techniques, The Human Embryo, Dr. Shigehito Yamada (Ed.), ISBN: 978-953-51-0124-6, InTech, Available from: http://www.intechopen.com/books/the-human-embryo/developmental-anatomy-of-the-human-embryo-3dimaging-and-analytical-techniques

\section{INTECH}

open science | open minds

\section{InTech Europe}

University Campus STeP Ri

Slavka Krautzeka 83/A

51000 Rijeka, Croatia

Phone: +385 (51) 770447

Fax: +385 (51) 686166

www.intechopen.com

\section{InTech China}

Unit 405, Office Block, Hotel Equatorial Shanghai

No.65, Yan An Road (West), Shanghai, 200040, China

中国上海市延安西路65号上海国际贵都大饭店办公楼 405 单元

Phone: +86-21-62489820

Fax: $+86-21-62489821$ 
(C) 2012 The Author(s). Licensee IntechOpen. This is an open access article distributed under the terms of the Creative Commons Attribution 3.0 License, which permits unrestricted use, distribution, and reproduction in any medium, provided the original work is properly cited. 